\title{
Atuação do enfermeiro na coleta do material cérvico-uterino
}

\author{
Nurse performance in the collection of the cervical-uterine material \\ Rendimiento de enfermeras en la recolección de material cervico-uterino
}

\begin{abstract}
RESUMO
Objetivo: analisar a atuação dos enfermeiros das unidades básicas de saúde na coleta do material cérvico-uterino. Método: trata-se de estudo de campo com abordagem quantitativa, realizado em março de 2019 em unidades básicas de saúde de um município paraibano. A amostra composta por 12 enfermeiros foi delimitada pelos critérios de inclusão: ser enfermeiro, atuar no serviço há no mínimo um ano e realizar a coleta do exame Papanicolau. 0 presente estudo obedeceu às normas e diretrizes regulamentadas pela resolução 466/12. Resultados: as principais dificuldades relatadas foram a realização da coleta do exame em mulheres menopausadas, obesas e gestantes. As profissionais expuseram que receberam capacitação, mas que a maioria não participou de eventos científicos nos últimos dois anos. Conclusão: observou-se a conduta e o perfil do enfermeiro na atuação da coleta do material cérvico-uterino, visto que a educação permanente precisa ser utilizada com uma estratégia de melhoria na atuação desses profissionais.
\end{abstract}

DESCRITORES: Enfermagem; Exame Papanicolaou; Câncer Uterino; Avaliação de desempenho; Consulta de Enfermagem.

\section{ABSTRACT}

Objective: to analyze the performance of nurses in basic health units in the collection of cervical-uterine material. Method: this is a field study with a quantitative approach, carried out in March 2019 in basic health units in a municipality in Paraíba. The sample composed of 12 nurses was delimited by the inclusion criteria: being a nurse, working at the service for at least one year and performing the Pap smear collection. The present study followed the rules and guidelines regulated by resolution 466/12. Results: the main difficulties reported were the collection of the exam in menopausal, obese and pregnant women. The professionals explained that they received training, but that the majority did not participate in scientific events in the last two years. Conclusion: the conduct and profile of the nurse in the practice of collecting cervical-uterine material was observed, since permanent education needs to be used with a strategy to improve the practice of these professionals.

DESCRIPTORS: Nursing; Pap smear; Uterine Cancer; Performance evaluation; Nursing consultation.

\section{RESUMEN}

Objetivo: analizar el desempeño de enfermeras en unidades básicas de salud en la recolección de material cérvico-uterino. Método: se trata de un estudio de campo con enfoque cuantitativo, realizado en marzo de 2019 en unidades básicas de salud de un municipio de Paraíba. La muestra compuesta por 12 enfermeras fue delimitada por los criterios de inclusión: ser enfermera, trabajar en el servicio durante al menos un año y realizar la recolección de Papanicolaou. El presente estudio siguió las normas y lineamientos regulados por la resolución 466/12. Resultados: las principales dificultades reportadas fueron la recolección del examen en mujeres menopáusicas, obesas y embarazadas. Los profesionales explicaron que recibieron formación, pero que la mayoría no participó en eventos científicos en los últimos dos años. Conclusión: se observó la conducta y el perfil del enfermero en la práctica de recolección de material cérvico-uterino, ya que la educación permanente debe ser utilizada con una estrategia para mejorar la práctica de estos profesionales.

DESCRIPTORES: Enfermería; Prueba de Papanicolaou; Cáncer uterino; Evaluación del desempeño; Consulta de enfermería.

RECEBIDO EM: 10/08/2020 APROVADO EM: 03/09/2020

\author{
Aline do Ó Araújo \\ Enfermeira pelo Centro Universitário de João Pessoa. \\ ORCID: 0000-0002-8257-3616
}


Thalys Maynnard Costa Ferreira

Enfermeiro pela UFPB. Mestre em Enfermagem pelo PPGENF/UFPB.

ORCID: 0000-0001-8758-6937

Rayza Régia Medeiros dos Santos de Oliveira

Enfermeira pela UFRN. Especialista em Enfermagem Obstétrica pela UFRN.

ORCID: 0000-0001-8725-6786

Danielly Gonçalves da Silva

Enfermeira pelo Centro Universitário Leão Sampaio. Especialista em Pediatria e Neonatologia pela FIP.

ORCID: 0000-0002-3525-0670

\section{Thaynara Ferreira Filgueiras}

Enfermeira pela UFCG. Mestra em Enfermagem pelo PPGENF/UFPB.

ORCID: 0000-0001-7520-4145

\section{INTRODUÇÃO}

0 Câncer de Colo de Útero é responsável pela segunda causa de morte entre mulheres por câncer no mundo, considerado um problema na saúde pública visto que, segundo os dados do Instituto Nacional do Câncer em 2016, são diagnosticados aproximadamente 500 mil casos novos por ano, e cerca de $80 \%$ desses casos são em países em desenvolvimento, no qual o Brasil está incluído. Sua maior incidência trata-se de mulheres com classe econômica mais baixa ${ }^{1}$.

$\mathrm{O}$ rastreamento das lesões precursoras do Câncer de Colo de Útero é um método de baixo custo, evidenciado por George Papanicolau, o exame citológico observa as alterações celulares provenientes de um raspado da cérvice, e entende-se que, foi adotado mundialmente para reduzir o desenvolvimento do câncer uterino e aplicado a protocolos bem definidos em programas de promoção e prevenção ${ }^{2}$.

O exame de Papanicolau deve ser realizado por profissionais de saúde, enfermeiros ou médicos, com formação e em capacitação contínua na atenção primária ${ }^{1}$. A resolução do COFEN no 381/2011 afirma que é privativo do enfermeiro a coleta do material para colpocitológia oncótica pelo método de Papanicolau, este procedimento deve ser executado no contexto da consulta de enfermagem, atendendo-se os princípios da Política Nacional de Atenção Integral a Saúde da Mulher ${ }^{3}$.

$\mathrm{O}$ rastreamento na atenção primária,
Eventualmente,

ocorrem casos

de mulheres

desenvolverem a

neoplasia no câncer

uterino, mesmo

realizando o exame

de citopatologia

periodicamente,

assim sendo $2 \%$

a $13 \%$ resultados

falso-negativo.

Estando relacionado

diretamente a

qualidade das coletas

do material citológico. na Estratégia da Saúde da Família, através da realização do exame citopatológico, em mulheres com faixa etária preconizada de 25 a 64 anos e/ou que seja caso de risco de acordo com o Ministério da Saúde, podendo levar a reduzir essas taxas de incidência e mortalidade da neoplasia em questão. $\mathrm{Na}$ fase inicial do câncer cervico-uteriro não há sintomas e as lesões precursoras podem ser detectada por meio do exame da citologia oncótica e tratada precocemente, com isso a possibilidade de cura em alguns casos são de até $100 \%{ }^{4}$.

Eventualmente, ocorrem casos de mulheres desenvolverem a neoplasia no câncer uterino, mesmo realizando o exame de citopatologia periodicamente, assim sendo $2 \%$ a $13 \%$ resultados falso-negativo. Estando relacionado diretamente a qualidade das coletas do material citológico. Fatores que levam a esse tipo de erro são a falta de atenção e concentração, a fadiga mental e a sobrecarga de trabalho ${ }^{4}$.

O presente estudo justifica-se pela necessidade imprescindível a capacitação dos enfermeiros que atuam na coleta na coleta do exame citopatológico, reforça-se o papel desse método na prevenção do câncer do colo do útero, e todos os esforços no sentido de reduzir a probabilidade de resultados incorretos devem ser incentivados, contribuindo, assim para a melhoria das condições de saúde. Tendo como questão norteadora do estudo: Qual a atuação dos enfermeiros em Unidades de Saúde da Família na consulta ginecológica na realização do exame citológico. 
Diante do exposto, o estudo teve como objetivo analisar a atuação dos enfermeiros em Unidades de Saúde da Família na consulta do exame Papanicolau em um município paraibano.

\section{MÉTODO}

Trata-se de um estudo de campo, descritivo, com abordagem quantitativa. O estudo foi realizado nas Unidades Básicas de Saúde do município de Lagoa Seca, Paraíba, em março de 2019. Estimou-se que aproximadamente no ano de 2017 uma população de 27.543 habitantes, onde possui 12 Unidades Básicas de Saúde, composta por 12 enfermeiros registrados nesses serviços 5 .

A população do estudo foi composta por todos os enfermeiros que atuam nas unidade de saúde da família e que realizavam a consulta de enfermagem na coleta do exame Papanicolau. Para a seleção da amostra foram considerados os critérios de inclusão: ser enfermeiro da atenção primária, atuando nas unidades há no mínimo um ano, na realização da consulta de enfermagem, na coleta do exame Papanicolau. Foram excluídos da amostra os enfermeiros que não atenderam a um ou mais dos critérios de inclusão ou que se recusaram a participar da pesquisa.

O projeto foi cadastrado na Plataforma Brasil e a coleta de dados realizada logo após a aprovação do projeto pelo Comitê de Ética em Pesquisa e da autorização da Secretaria Municipal de Saúde, concretizada no mês de março de 2019. Inicialmente foi realizada uma breve explanação sobre os objetivos da pesquisa aos participantes da pesquisa, no intuito de conseguir uma maior aceitação na participação do estudo. Uma vez dispostas a contribuir com o estudo, o participante assinava o Termo de Consentimento Livre e Esclarecido e era iniciada a aplicação dos questionários. $\mathrm{O}$ instrumento de coleta constituiu em um roteiro de um questionário estruturado, elaborado pela própria pesquisadora, composto por duas partes: a primeira tratava-se do perfil sócio demográfico e identificação dos enfermeiros e a

\section{O projeto foi}

cadastrado na

Plataforma Brasil

e a coleta de dados

realizada logo

após a aprovação

do projeto pelo

Comitê de Ética

em Pesquisa e

da autorização

da Secretaria

Municipal de Saúde,

concretizada no mês

de março de 2019. segunda composta por questões em torno dos objetivos do estudo.

No processo de digitação o banco foi digitado aos pares com o intuito de evitar possíveis erros na codificação das variáveis e preenchimento do banco.

A pesquisa foi realizada obedecendo todas as normas éticas regidas pela Resolução 466/2012 e aprovado com número de aparecer CAAE 07234819.7.0000.5176.

\section{RESULTADOS E DISCUSSÃO}

A amostra desse estudo foi composta por $100 \%(n=12)$ profissionais graduadas em enfermagem, na sua totalidade do sexo feminino e atuantes em unidades básicas de saúde do município de Lagoa Seca. A idade com maior representação foi entre 30 a 59 anos que correspondiam a $75 \%(n=9)$, $8,3 \%(n=1)$ adultos jovens e $16,7 \%(n=2)$ eram idosas com 60 ou mais anos. Na variável estado conjugal existiu uma prevalência de mulheres casadas, correspondiam a $66,7 \%(n=8), 50 \%(n=6)$ eram católicas e $33,3 \%(n=4)$ evangélicas. No que se refere ao grau de escolaridade, $50 \%(n=6)$ eram especialistas, seguido $33,3 \%(n=4)$ da titulação de mestre. Quanto ao item "Participação em eventos”, $58,3 \%(\mathrm{n}=7)$ participantes relataram não participaram de eventos nos últimos dois anos.

Em relação aos passos seguidos na coleta do exame cérvico-uterino, é recomendado que o profissional deve seguir os passos descritos no questionário: identificação, informações sobre o exame, histórico clínico, preenchimento do formulário e preparação da lâmina, destaca-se que no estudo dentre os profissionais participantes, duas enfermeiras não seguiam as etapas recomendadas ${ }^{10}$.

Quanto ao preenchimento correto da requisiçã̃o, o histórico clínico e os dados completos da paciente são passos fundamentais para uma boa qualidade no resultado do exame. Em outras pesquisas realizadas demonstram que todas as entrevistadas preenchiam o formulário em sua totalidade ${ }^{11}$.

Nas principais dificuldades durante a realização da coleta de material cérvico uterino, as mais apontadas são mulheres meno- 


\section{artigo}

Araújo, A.O.; Ferreira, T.M.C.; Oliveira, R.R.M.S.; Gonçalves da Silva, D.; Filgueiras, T.F.

Atuação do enfermeiro na coleta do material cérvico-uterino

pausadas, obesas e gestantes. Em pesquisa realizada ${ }^{12}$ em São Miguel do Oeste com enfermeiros atuantes em unidade básicas de saúde, esses relataram que as dificuldades apresentadas eram a execução do exame em mulheres obesas, com limitações físicas, vergonha durante o exame, resistência das mulheres, dentre outras.

Dados da pesquisa demonstram que a maioria das entrevistadas afirmaram não

\section{Tabela 1 - Roteiro de entrevista. João Pessoa, Paraíba, Brasil, 2019. (N=12)}

Variáveis

n

$\%$

Quais os passos da coleta?

(1)Identificação (2) Histórico (3)Informações (4) Preenchimento das lâminas (5)Preenchimento do formulário

Todas as alternativas

10

Menos a alternativa 5

Menos a alternativa 4

Quando observado alterações na realização do exame onde é realizado o registro?

Prontuário, requisição de exames e livro de registro

Prontuário e requisição de exames

Prontuário

Após registros essas informações foram enviadas ao laboratório?

Sim

Não

Preenchimento do formulário da citologia para requisição do exame citopatológico

Sim

Não

É notório amostras insatisfatórias no resultado

$\operatorname{Sim}$

Não

O instrumento preenchido conduz pontos abordados durante a consulta de enfermagem

$\operatorname{Sim}$

Não

Fonte: Dados da Pesquisa, 2019

Tabela 2- Relato das dificuldades na realização do exame cérvico-uterino. João Pessoa, Paraíba, Brasil, 2019. (N=12)

Variáveis

Não apresentou dificuldade $\%$

Mulheres menopausadas, obesas, gestantes

Medo da paciente na realização do exame

Ausência da DUM

Visualização do Colo uterino

18,3

Demora na entrega do resultado do exame

Fonte: Dados da Pesquisa, 2019 identificar quando a amostra é insatisfatória, avaliando também se o material é enviado corretamente para o laboratório, todas acondicionam e acompanham a requisição à lâmina, dados que corroboram com estudo realizado $^{13}$, que referiam que as três causas mais frequentes de amostras insatisfatórias foi a presença de material em menos de $10 \%$ do esfregaço, a ausência ou erro na identificação da lâmina, frasco ou formulário e a presença de artefatos de dessecamento.

Diante dos resultados desse estudo observa-se a contribuição da pesquisa para intensificação do monitoramento do câncer do colo uterino, visto que faz-se necessário a qualificação dos profissionais para realização de consulta de enfermagem com excelência, ampliando o número de exames preventivos com um resultado mais fidedigno.

O exame citopatológico é parte do processo de rastreamento, e vários são os fatores que podem estar relacionados ao insucesso de um programa como um todo. Esses fatores existentes são fundamentais com bastante importância para que a qualidade da análise dos exames citopatológicos não seja comprometida ${ }^{14}$.

Em estudo realizado ${ }^{15}$ ressalta que são fundamentais as orientações, aumentando a compreensão das mulheres sobre a importância da prevenção do câncer de colo uterino e do autocuidado, proporcionando-lhes espaço para refletirem sobre a prevenção do câncer cervico uterino, e procurando, por meio de atividades educativas, esclarecê-las da importância do autocuidado para reduzir os fatores de risco predisponentes ao surgimento do câncer e otimizando o desenvolvimento de políticas de prevenção. Essa mesma estratégia é utilizada nesse estudo em que enfermeiras afirmam orientar a população sobre promoção e prevenção da saúde.

No presente estudo os profissionais em maiores percentuais afirmaram que não receberam capacitação para realização do exame citopatológico, o que assemelha com achados de outros estudos que foram observadas lacunas no processo de capacitação profissional periodicamente ${ }^{16}$.

Ao finalizarmos esse estudo observa- 
-se a existência de falha no processo de capacitação permanente de profissionais na saúde pública, o problema agrava-se na constatação de dados em que os enfermeiros das UBS não aplicam estratégias de prevenção e controle do câncer uterino por falta de capacitação na área ${ }^{17}$. Nesse contexto, que ocasiona a inexatidão no processo do exame do Papanicolau.

\section{CONCLUSÃO}

Em virtude dos resultados mencionados, percebe-se que ainda existem alguns elementos dificultadores do próprio sistema de saúde. O câncer do colo do útero é um problema da saúde pública, responsável pela segunda causa de morte entre mulheres por câncer no mundo.

Esse estudo mostra que não é só a falta de interesse da população alvo na realização do exame Papanicolau, os profissionais de saúde, em especial, o enfermeiro é participante da não execução e quando executado o exame citológico esse não segue as recomendações da instâncias, servindo de exemplo para outros profissionais à importância que tem uma simples falha na etapa, pode omitir no resultado do exame citopatológico.

A educação permanente precisa ser utilizada com uma estratégia de melhoria na atuação desses profissionais, atentando que enfermeiros qualificados resultam em um rastreamento de excelência do câncer uterino.

Sugere-se que novas discussões e estudos sejam realizados com ênfase no que a população diz sobre o exame do Papanicolaou e atuação dos enfermeiros em maior abrangência, em outros municípios paraibanos, em outras realidades. -

\section{REFERÊNCIAS}

1.Silva LR, Almeida CAPL, Sá GGM, Moura LKB, Araujo ETH. Educação em saúde como estratégia de prevenção do câncer do colo do útero: revisão integrativa. Revista Prevenção de Infecção e Saúde. 2018;3(4):35-45.

2.Mello FA; Galle LC, Prado RL. Prevenção do câncer de colo uterino na concepção da população feminina de uma cidade do interior do estado de São Paulo. Colloquium Vitae.2018,9(2):45-52.

3.Conselho Federal de Enfermagem. Resolução COFEN $n^{\circ}$ 381/2011. [Internet]. Available from: http://novo.portalcofen. gov.br/resoluo-cofen-n-3812011_7447.html

4.Ministério da Saúde. Instituto Nacional de Câncer. Coordenação de Prevenção e Vigilância. Divisão de Detecção Precoce e Apoio à Organização de Rede. Manual de gestão da qualidade para laboratório de citopatologia. 2. ed. rev. ampl. Rio de Janeiro, 2016.

5.Instituto Brasileiro de Geografia e Estatística. Diretoria de Pesquisas, Coordenação de População e Indicadores Sociais, Estimativas da população residente com data de referência. Available from: https://www.ibge.gov.br/cidades-e-estados.ht$\mathrm{ml}$ ?view=municipio

6.Filgueiras TF, Silva RA, Pimenta CJL, Filgueiras TF, Oliveira SHS, Castro RCMB. Instrumento para consulta de enfermagem a gestantes com diabetes mellitus. Revista da Rede de Enfermagem do Nordeste. 2019, (20).

7.Ribeiro GC, Santos FEC, Francalino TR, Mendes IC. Utilização de tecnologias de promoção da saúde pelos enfermeiros na atenção primária. Encontro de Extensão, Docência e Iniciação Científica (EEDIC), [S.I.], v. 5, n. 1, 2019. Available from: http:// publicacoesacademicas.unicatolicaquixada.edu.br/index.php/ eedic/article/view/3148/2697

8.Araujo MAN, Lunardi Filho WD; Alvarenga MRM, Oliveira RD, Souza JC; Vidmantas, S. Perfil sociodemográfico dos enfermeiros da rede hospitalar. Revista de Enfermagem da UFPE , 2017,11(11):4716-25.

9.Becker RM, Heidemann ITSB, Meirelles BHS, Costa MFBNA, Antonini FO, Duran MK. Nursing care practices for people with Chronic Non communicable Diseases. Revista Brasileira de En- fermagem, 2018,71(6):2643-9.

10.Ministério da Saúde. Secretaria de Atenção à Saúde. Departamento de Atenção Básica. Controle dos cânceres do colo do útero e da mama / Ministério da Saúde, Secretaria de Atenção à Saúde, Departamento de Atenção Básica.2. ed. Brasília : Editora do Ministério da Saúde, 2013.

11. Mendes YLC, De Mesquita KO, Lira RCM. Prevenção do câncer de colo uterino: analisando a atuação do enfermeiro da atenção primária à saúde. SANARE-Revista de Políticas Públicas, $2015,14(2)$.

12.Souza SQF; Bauermann KB. Dificuldades encontradas pelos enfermeiros na realização da coleta de material cérvico-uterino que dificultam ou inviabilizam o exame Papanicolau [TCC], São Miguel do Oeste, 2016.

13. Galvão EFB, Silva MJM, Esteves FAM, Peres AL. Frequência de amostras insatisfatórias dos exames preventivos do câncer de colo uterino na rede pública de saúde, em município do agreste pernambucano. Revista Paraense de Medicina, 2015,29(2):51-6.

14. Instituto Nacional de Câncer José Alencar Gomes da Silva. Coordenação de Prevenção e Vigilância. Divisão de Detecção Precoce e Apoio à Organização de Rede. Manual de gestão da qualidade para laboratório de citopatologia, Divisão de Detecção Precoce e Apoio a Organização de Rede. 2. ed. rev. ampl. Rio de Janeiro, 2016. $160 \mathrm{p}$.

15. Andrade CB, Souza C, Campos NPS, Gonzaga MFN, Pereira RSF, Soares APG. Percepção dos enfermeiros da atenção básica à saúde do município de Jeremoabo frente à resistência das mulheres na realização do Exame Citopatológico de Colo de Útero. Revista Saúde em Foco, 2017, 9:34-55.

16. Moraes DC, Almeida AM, Figueiredo EN, Loyola EAC, Panobianco MS. Opportunistic screening actions for breast cancer performed by nurses working in primary health care. Revista Escola de Enfermagem da USP. 2016;50(1):14-21.

17.França, T. F. Atenção burocrática na saúde da mulher: prevenção e rastreamento de câncer de colo de útero pelo enfermeiro. [Dissertação], 2016. 